\title{
Design and applications of flexible photonic membranes
}

\author{
Peter Reader-Harris, Blair C. Kirkpatrick and Andrea Di Falco \\ SUPA, School of Physics and Astronomy, University of St Andrews, North Haugh, St \\ Andrews, KY16 9SS, United Kingdom
}

\begin{abstract}
In this paper we present the design and applications of flexible photonic membranes. We discuss their use as versatile photonic layers in the framework of lab-on-fibre applications, specifically focusing on the design of angular robust spectral filters. We also show alternative routes to their fabrication, highlighting the opportunities and limitations associated to each approach. Finally we present our preliminary results on the all-optical control over flexible membranes, as a potential method to fine-tune their opto-mechanical properties to the required application.
\end{abstract}

Keywords: flexible plasmonics, optical tweezers, lab-on-fibre

\section{INTRODUCTION}

Flexible photonics is a burgeoning field which promises to revolutionise many applications based on the manipulation of light, including flexible displays, ${ }^{1}$ electronic paper $^{2}$ and flexible solar cells. ${ }^{3}$ Recently, flexibility has been introduced into the traditionally flat and rigid world of nanophotonics, where matter needs to be shaped at a length scale much smaller than the operating wavelength. ${ }^{4,5}$ The ability to modify the shape of the substrate offers post-fabrication tuning possibilities ${ }^{6}$ and allows complex shaped objects to be functionalized. ${ }^{7}$ In this framework, we have recently developed the concept of Metaflex, flexible metasurfaces ${ }^{8,9}$ in the optical range of frequencies. ${ }^{10}$ The versatility offered by the nanoplasmonic layer has been exploited to design and fabricate devices with diverse optical functions, including field enhancement, ${ }^{10}$ negative index materials ${ }^{11}$ and metasurfaces with engineered reflectivity. ${ }^{12}$

In this paper we first revise, in section 2, the available fabrication protocols for nanoplasmonic photonic membranes, highlighting the advantages and limitations of each approach.

The ability of these membranes to conform to target objects is discussed in section 3 in the context of labon-fibres applications. This method permits the transfer of arbitrarily complex photonic functions onto the tip of optical fibres. Our approach removes the fabrication requirements typically associated with directly writing features onto the fibre facet. Specifically we discuss a design of spectral filter that is compatible with the relatively high numerical aperture of standard fibres. Our design allows the placement of a lightweight, ultrathin photonic membrane (less than $200 \mathrm{~nm}$ thick) over the end of the fibre in a removable fashion. As lab-on-fibre matures into an end-user technology, the ability to use a single fibre for light delivery, backed by a light source and detector, which can be functionalized in different way by simply replacing the fibre termination cap, will greatly simplify the ease of use.

Finally, in section 4 we present our preliminary results on the development of an all-optical approach to the precise manipulation of the photonic membranes. Much literature concerned with probing the mechanical properties of membrane-like structures is based upon first attaching the membrane to some form of dielectric sphere "handles". These handles are then trapped and can be used to move and warp the membrane with relative control. ${ }^{13}$ We aim to achieve this same degree of control, not by employing the additional step of attaching dielectric beads to the photonic membrane, but rather through the design process of the membrane itself. This method promises to drastically improve the accuracy of the placement of the photonic layer with respect to a target object, but most importantly it lays the basis for an experimental platform to dynamically address the opto-mechanical response of the membranes.

Further author information: (Send correspondence to A.D.F.) A.D.F.: E-mail: adf10@st-andrews.ac.uk, Telephone: +44 (0)1334 463165, Website: www.st-andrews.ac.uk/physics/synthopt

Quantum Sensing and Nanophotonic Devices XI, edited by Manijeh Razeghi, Eric Tournié, Gail J. Brown, Proc. of SPIE Vol. 8993, 89930P - (c) 2014 SPIE · CCC code: 0277-786X/14/\$18 · doi: 10.1117/12.2039139 


\section{FABRICATION}

Fabrication of flexible photonic membranes proceeds along a number of routes. In this section we present three complementary ways, etch back, self-assembly monolayer templating and metallic ink printing.

The etch back is a top down process, as sketched in figure 1a). The key feature which makes this technique notable for the fabrication of flexible photonic membranes is that it uses only standard techniques on planar substrates, until the final release step. Two polymers are spun on top of a rigid substrate, typically silicon. The first is the sacrificial layer (Microchem Omnicoat), which is later removed by a solvent in order to detach everything above from the silicon. The second layer is the membrane layer, for which we use Microchem SU8, an epoxy based polymer which is extremely resistant to all the other processes used in this protocol. After spin coating the membrane layer is induced to polymerize by $30 \mathrm{sec}$ exposure to ultraviolet light and heating at 100C for $5 \mathrm{~min}$, before a layer of gold of the required thickness is deposited via electron beam evaporation. A photoresist layer is then spun on top, for which we use SU8 as negative tone resist for electron beam lithography, or ZEP520A, which is a positive tone resist, depending on the pattern's requirement. The resist is then patterned and developed in ethyl lactate solvent (SU8) or xylene (ZEP) and transferred on to the gold with Argon based reactive ion etching. This leaves the desired metallic pattern on top of the membrane, which can then be released by dissolving the initial sacrificial layer with $N$-methyl-2-pyrrolidone (NMP). Once the membrane is left suspended in the solvent, it is often convenient to transfer it by scooping into another liquid bath. ${ }^{14}$ SU8 is strongly hydrophobic, thus it tends to float on the surface of water, enabling easy transfer onto a frame or new substrate. Typical membrane fabricated with this approach have thicknesses of $4 \mu \mathrm{m}$, down to less than $200 \mathrm{~nm}$, a gold thickness of $40 \mathrm{~nm}$ and feature sizes of a few 10 s of nanometers.

An alternative fabrication approach is based on Self-assembly monolayers (SAMs) templates. Aromatic SAMs, e.g. of $\omega$-(4'-methyl-biphenyl-4-yl)thiol $\left(\mathrm{CH}_{3} \mathrm{C}_{6} \mathrm{H}_{4} \mathrm{C}_{6} \mathrm{H}_{4} \mathrm{SH}, \mathrm{MBP} 0\right)$, have a positive resist-type behaviour, where an electron beam can locally induce crosslinking. ${ }^{15}$ This effect can be used to create a template for the growth of metallic features, which can then be electrochemically deposited onto the substrate onto which the SAM is deposited. ${ }^{16}$ Figure 1b) shows the scheme. First a SAM is created on the gold substrate via immersion in solution. The thiol group at one end of the molecule has a strong affinity for the gold substrate, and so a monolayer is formed, as shown in figure 1b). After local crosslinking by electron beam exposure, copper ions are deposited by electrodeposition in the unexposed SAM regions. The copper can be subsequently peeled off via application of a flexible substrate. This final process is aided by the mushroom-like shape of the copper formation in the SAM, reducing the contact area of the deposited metal on the metal substrate, and the fact that Copper has a low adhesion onto the SAM. This templating technique is particularly suited to reusability, because the expensive patterning of the SAM template needs to be done only once. Using this approach it is possible to define metallic features down to few tens of nanometers. Further experiments are in progress to deposit gold rather than copper, for improved plasmonics behaviour.

The third and last fabrication example presented is based on the transferring of plasmonic nano features via stamping of metallic ink, ${ }^{17}$ as shown in figure 1c). First a mould template is fabricated using standard top down fabrication techniques, in this case electron beam lithography of SU8 on silicon followed by development. A Polydimethylsiloxane (PDMS) stamp is then made by casting it into the mould, and allowed to cure. A monolayer of metallic nanoparticles kept in a chloroform solution is then formed on the surface of a water bath. The PDMS stamp is then inked by moving the stamp down onto the nanoparticles suspension. Before the inking, the PDMS stamp is treated with fluorinated silane to reduce the strength of the particle adhesion and promote the transferring on the target substrate. The two main appealing features of this approach are scalability and conformability of the stamp. Once the stamp has been fabricated the inking and imprinting procedure can be repeated several time, or even mounted on a printing drum, allowing for a reusable and highly scalable fabrication route for flexible plasmonic structures. Additionally, the polymeric stamp can be made very flexible to adapt to complex target topologies. This enables the definition of metallic nano features on samples of virtually arbitrary shape and material, which could not be written with traditional techniques requiring planar targets, like electron beam lithography. The main limitation of this approach is the care required to obtain connected plasmonic 


\section{a) Etch back \\ Spin coat \\ b) $\begin{gathered}\text { Self assembly } \\ \text { monolayer }\end{gathered}$ templating} sacrificial layer

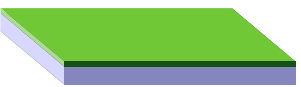

\section{Spin coat} membrane

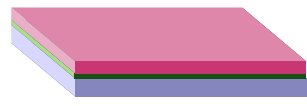

Evaporate gold
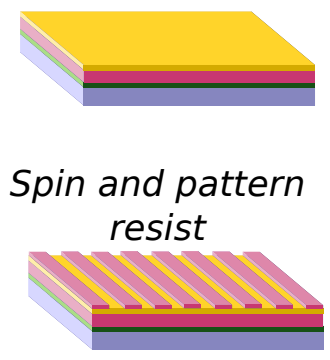

Etch pattern

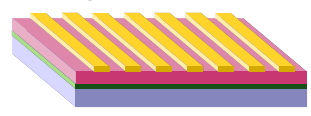

Release membrane

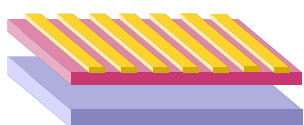

SAM grown on gold

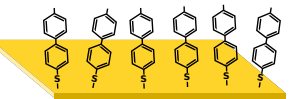

Irradiation selectively crosslinks molecules

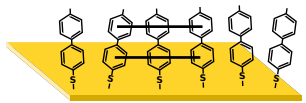

Copper deposition where not crosslinked

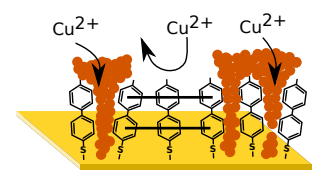

Application of flexible substrate

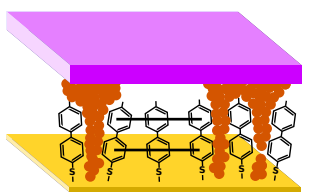

\section{Substrate removed} with pattern

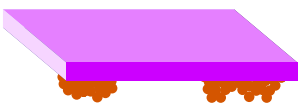

\section{C) Metallic ink printing}

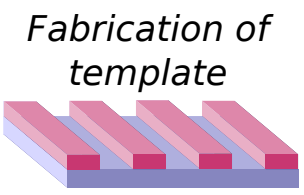

Casting of PDMS mold

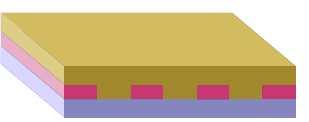

Single layer of nanoparticles on water ink mold

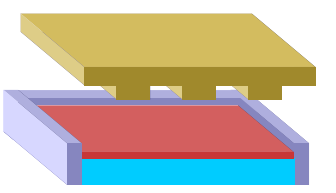

\section{Stamping onto} flexible susbtrate

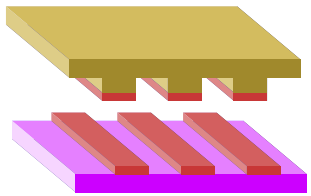

Figure 1. a) A typical scheme to fabricate flexible photonic membranes using the etch back method. Layers are built up, before a top protective layer is patterned and the whole sample is etched from the top. The first layer is dissolved to leave a flexible membrane behind.

b) SAM templating. A SAM is grown and selectively cross linked using e-beam lithography. Copper is then electrochemically deposited, and can seed where the SAM was not irradiated. An adhesive flexible substrate can then lift off the copper structures, breaking them at a weak point in their "mushroom" shape.

c) Fabrication steps to realise metallic ink printing. A template is fabricated using any top-down method, such as in a). A PDMS mold is then cast and inked with nanoparticles from a monolayer suspension in water. This is then stamped onto a flexible substrate. 
a)

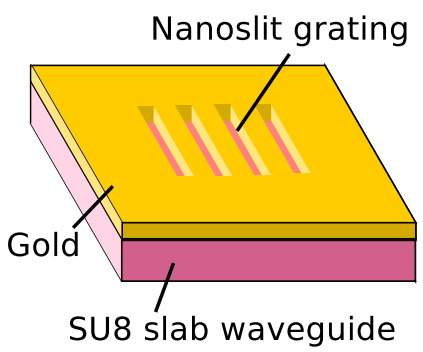

b)

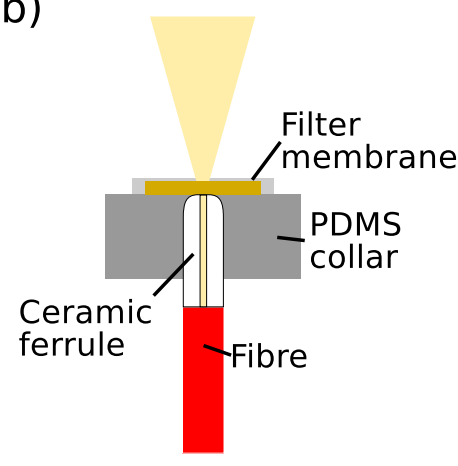

c)

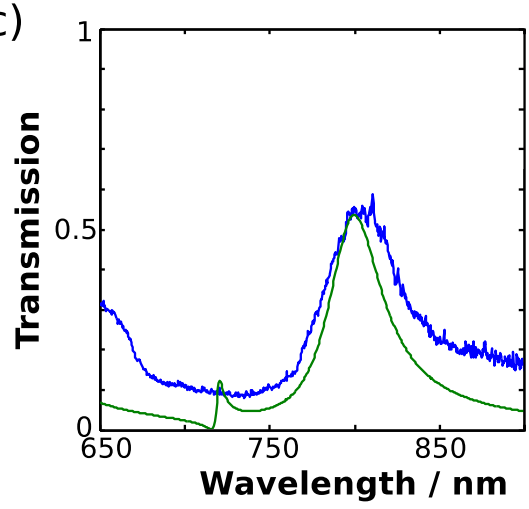

Figure 2. a) A schematic of the nanoslit structure of the filtering membrane b) A schematic of the fibre termination assembly c) A comparison of the RCWA curve at normal incidence (green) and the spectrum from the membrane terminated fibre experiment

elements, as the ligands around the nanocolloidal particles prevents adjacent nanoparticles coupling to form extended plasmonic surface waves.

\section{LAB-ON-FIBRE}

The nanophotonic membrane paradigm could be advantageous for the emerging field of lab-on-fibre technology. This approach is the fibre-based analog of the lab-on-chip concept, and merges the light delivery capabilities of fibre optic cable, with the functionalities of photonic crystals and metamaterials. ${ }^{18}$ The two alternative fabrication routes available are direct writing on fibre facets and the application of a functionalized surface to fibre. The direct writing approach is based on traditional lithographic techniques, e.g. electron beam lithography or focused ion beam etching. This is the most straightforward way to define nano features with high degree of control and versatility. However, these methods require advanced manipulation of the fibres, e.g. to spin coat their tips and to position and align them with the accuracy required by the writing process. With the functionalized surface approach on the other hand the photonic layer is fabricated independently and further applied to the tip of the fibre. This decouples the choice of the fibre from the fabrication of the photonic element, leading to a much simpler way of realizing the desired function. Rigid photonic layers ${ }^{19}$ have been successfully transferred on the tip of fibres.

This approach has the additional advantage that the fibres are not permanently modified. Membranes could be mounted into fibre terminating packages and simply replaced by the end user, depending on the experiment being performed. This allows one fibre and sensing apparatus to be used for a variety of different measurements. The mechanical and optical properties of flexible polymeric membranes makes them versatile alternative to their rigid counterparts.

The simplest example of a useful functional ending to a fibre is a spectral filter. Recently, we demonstrated the fabrication of a flexible filter based on guided mode resonances (GMR) mounted onto a aluminium foil frame, wrapped over the end of a fibre terminated with a compact collimating package. ${ }^{20}$ Here we demonstrate a filter design that eliminates the need for the collimator, by creating an angularly robust filter.

The GMR filter was proposed as method to create filters by using subwavelength gratings to selectively couple light into a waveguide. ${ }^{21}$ Figure 2a) shows a schematic of the type of system required, etching nanoslits into a gold film on top of a waveguiding layer. The modes which the waveguide/gold layers can support determine the filtering action. We use SU8 as our dielectric slab waveguide and gold as our metallic layer, as exemplified in figure 1a). By performing Rigorous Coupled Wave Analysis (RCWA $)^{22}$ simulations we have found that this arrangement is a versatile way of making a variety of different filters, by choosing suitable geometrical parameters. By changing the duty cycle it is possible to design a notch filter in transmission (low duty cycle), or a passband filter (high duty cycle), with opposite behaviour of the reflection. The absorption can also be controlled to play 
a more or less significant role, e.g. tuning the thickness of the metal, while the period choice determines the frequency position of the the spectral features.

GMR filters are typically very sensitive to the incident angle of light. Various methods have been proposed to create more angularly robust GMR filters. These techniques typically involve controlling the bandstructure by using multi-period gratings, ${ }^{23,24}$ although at a cost of additional fabrication complexity. Exploiting the anticrossing of the plasmonic modes, rather than those of the waveguide, we demonstrate here an angularly robust filter. The waveguide modes experience only a small change in energy owing to degeneracy lifting, but the plsamonic modes are much more strongly confined and the geometry of the system is such that the degeneracy lifting causes much larger energy shifts. This leads to a much stronger anti-crossed repulsion of the bands. By anti-crossing the modes at the $\Gamma$-point of the Brillouin Zone, we achieve angular robust filtering around normal incidence. It should be noted that typically this comes at the cost of the narrowness of the spectral features, because metals have an associated loss which reduces the quality factor of their resonances.

Initially, after the photonic membrane was released (following the final step in figure 1a) it was hydrophobically suspended on the surface of water so that the membrane could be mounted onto a frame. Subsequently the membrane was transferred onto a PDMS collar for fibre mounting. The flexibility of the sample makes this transfer process very easy, and enables good adhesion to the target mount, as shown in figure $2 \mathrm{~b}$ ). The fibre cores are typically only a few 10s or microns across, and so filters of the required size can easily be fabricated without recourse to large area lithographic techniques.

At one end, the fibre was terminated with the PDMS collar and the filter membrane. Broadband light from an ocean optics halogen lamp was coupled to the other end of the fibre with a standard launch platform. The filtering membrane was placed on the PDMS holder with a micropositioner stage, to align the $50 \times 50 \mu \mathrm{m}$ filtering area over the $5 \mu \mathrm{m}$ diameter fibre core. Both imaging and spectral measurements were performed with a $10 \times$ objective, with a working distance of $33.5 \mathrm{~mm}$. Figure 2c) shows a typical measured filtered spectrum, and the spectrum calculated at normal incidence using RCWA, without any fitting parameters. The extra resonance at around $720 \mathrm{~nm}$ which can be seen in the simulation is due to a waveguiding resonance with the polarization such that the electric field is parallel to the nanoslits (TE). This does not show in the experimental data because it is highly angularly dependant, as opposed to the TM plasmonic resonance (electric field perpendicular to the nanoslits), and so is smeared out by the fibre. Off-resonance the filter prevents the transmission of TE light, less than $10 \%$ across the spectrum shown). A good agreement can be seen, showing that the filter does indeed perform the same filtering action over the whole range of angles which the fibre, with an NA of 0.14 , emits. The grating structure also provides polarisation control of the fibre output, although only the unpolarised spectrum is shown here.

This demonstrates the utility of the flexible photonic membrane, as it shows that an ultra-thin membrane can be used for filtering by direct application to a fibre facet. This is a lightweight and reusable alternative to direct writing techniques, which can make use of all the formalisms introduced to describe metasurfaces and plasmonic waveguides, providing a testbed for further lab-on-fibre technologies.

\section{OPTICAL MANIPULATION OF PHOTONIC MEMBRANES}

An accurate manipulation of the photonics membranes can be achieved with mechanically actuators down to the micron range, as for example required to align the membranes on the tip of fibres. However, to unlock the full tuning potential of the nanoplasmonic devices we are developing an all-optical actuation platform. To this extent, we utilise an inverted optical tweezers system as shown in figure 3. A diode-pumped solid state laser $(\lambda=830 \mathrm{~nm})$ is shone first through an expanding telescope and then a relay arm, before being focussed onto the sample by a $\times 60$ objective lens (Olympus UPLSAPO 60XW, NA =1.2). The use of dichroic mirror and infrared filter allows the user to decide whether or not a small reflection of the laser light from the sample can be projected onto the camera. In this way one may position the optical traps as desired, then place the filter in the path of beam, allowing particle behaviour to be observed without the signal to the camera being dominated by the light from the laser. The high numerical aperture of the objective lens ensures that the light field in the sample plane exhibits very strong intensity gradients around any and all foci. It is these extreme intensity gradients that give rise to the trapping force; thus particles which are close enough to "feel" this gradient are 


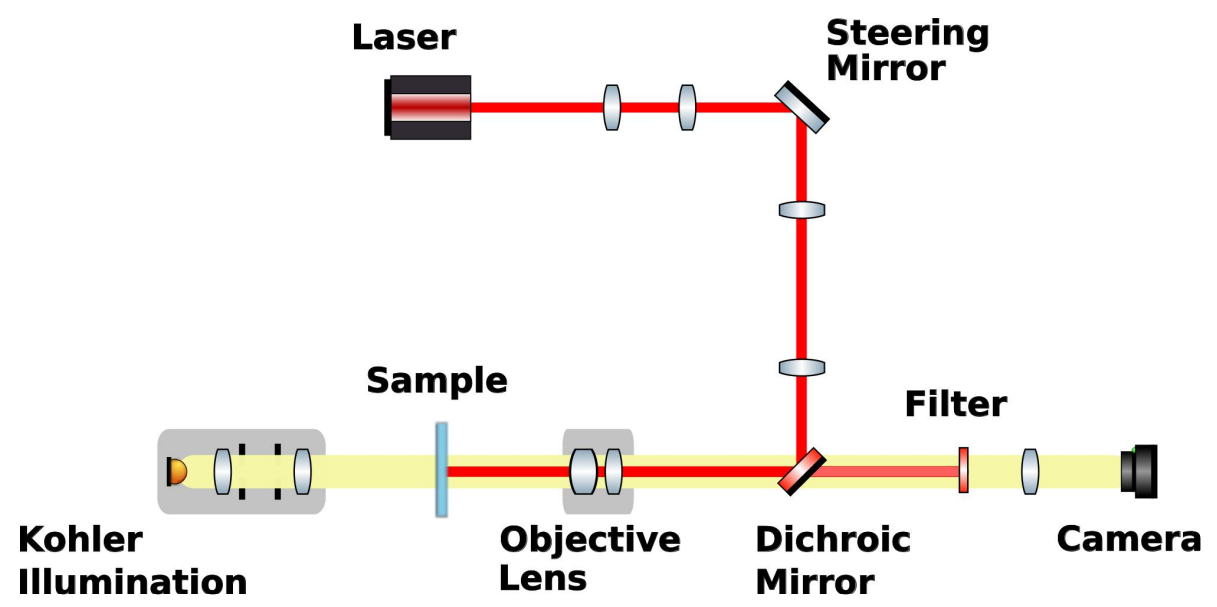

Figure 3. Schematic diagram highlighting the components used in a typical optical tweezers scheme. An objective lens acts both to focus the laser into a sharp focus, creating an optical trap, and to image the sample onto the CCD camera. The relay arm, consisting of a steering mirror and telescope, ensures that any tilt in the steering mirror results in an equivalent translation of the laser focus in the plane of the sample.

pulled in towards the beam's focus, and are trapped there. In addition to feeling a translational "pull" due to the trapping beam, any trapped object will experience a torque imparted on it by the beam. For the case of rotationally symmetric particles, this optical torque is inconsequential as far as the orientation of the particle is concerned. However, if a rotationally non-symmetrical particle (for example, a membrane) is trapped, then the effects of optical torque become important. Roughly speaking the trapped object will be reoriented such that its longest axis is aligned along the optical axis of the laser. This orientation represents the lowest energy state of the system.

In the following we present our preliminary results on the optical trapping of polymeric membranes.

Figure 4a)-c) show the behaviour of a $5 \mu \mathrm{m} \times 5 \mu \mathrm{m}$ wide and $600 \mathrm{~nm}$ thick SU8 samples. The panel (a) shows the membrane in its equilibrium position, lying perpendicular to the optical axis of the trap, when the laser is turned off. With the laser turned on, the membrane can be seen to flip (b) such as to align its longest axis with that of the optical axis. This "flipping" typically occurs over the course of 3-4 seconds. If the laser is then subsequently turned off as in (c), the membrane slowly returns to its equilibrium position over the course of roughly 10-12 seconds. This flipping motion is brought about by the optical torque produced by the tweezers. ${ }^{25}$ The membrane can be moved through the sample by the trap in its "flipped" orientation.

If required by the specific application, the response to the trap can be controlled by tailoring the geometry of the membrane, e.g. to stabilise its trapped position transversally to the beam. It is also possible to frustrate the tilting of the membrane by anchoring it to an extended support. Figure 4d)-f) shows the response of another set of $5 \mu \mathrm{m} \times 5 \mu \mathrm{m}$ wide membranes, this time of thickness $3 \mu \mathrm{m}$, connected to each other by a $600 \mathrm{~nm}$ thin SU8 layer. The overall membrane covers an area of $\sim 100 \mu \mathrm{m} \times \sim 100 \mu \mathrm{m}$. Figure $4 \mathrm{~d}$ ) shows a membrane reacting to an optical trap placed a few microns away. (e) The membrane has been dragged in by the trapping beam such that the edge of the membrane is now at the focal point of the trap. (f) the membrane reaches a point were it is stably trapped. Here there is no flipping observed, due to the large transverse extent of the membrane, although the membrane can still be coaxed into moving laterally with the trapping beam. On one hand this experiment demonstrates that the trapped position of a single membrane can be effectively stabilised by tweaking its topology. On the other hand it show that it is possible to design and manipulate extended objects, which is interesting for complex optical functions. It should be noted that any lateral control over the membrane's position is significantly weakened, and that the response of the membrane to changes in the position of the beam is weaker than for the single membrane. This is no doubt caused by the increased bulk of the target membrane. For most purposes, the desired system would have behaviour somewhere in between the two regimes discussed. Thin, manoeuvrable membranes could be fabricated with thicker sections or "handles" which would 

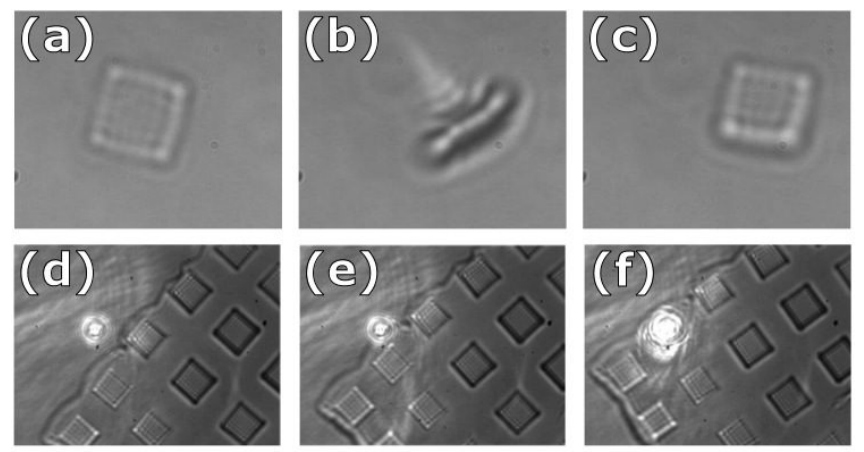

Figure 4. Influence of the trapping beam on simple $600 \mathrm{~nm}$ thick polymeric membranes. Top row shows "flipping" behaviour exhibited by a $5 \mu \mathrm{m} \times 5 \mu \mathrm{m}$ SU8 membrane (note that the laser light itself cannot be seen, as an IR filter was used to block the beam before it could reach the camera). A larger membrane, with an edge of length of $\sim 100 \mu$ m, is shown in the bottom row of figures. It does not display the "flipping" phenomenon, but its increased bulk makes it much harder to move around within the sample. Image credit: Mathivanan Damodaran.

provide locations for stable trapping to occur. These handles can then be used to pull the membrane through the sample, while limiting light exposure to those parts of the membrane that are partial to flip.

Further improvements can be made by controlling not only the physical dimensions of the membrane, but also the intensity distribution of the light that is incident upon it. This can be achieved through use of a diffractive optical element (DOE), which can create a particular distribution of light in the plane of the sample, as determined by the user. This DOE, typically a spatial light modulator (SLM) is placed in a plane conjugate to the back focal plane of the objective lens. The resulting intensity distribution of light in the sample is then merely a scaled Fourier transform of the phase pattern displayed on the SLM. ${ }^{26}$ In this way, the user is capable of realising several trapping beams in the sample plane, each of which can be moved completely independently of the others. This "multiple tweezers" approach provides a further level of control and suggests a route to control photonic membranes with the desired precision. ${ }^{27}$

\section{CONCLUSION}

Flexible photonic materials will continue to provide a useful way of bringing the paradigms of plasmonics and metasurfaces to a new range of applications. We have demonstrated that the conformability of a flexible nanoslit grating, acting as a GMR filter, can provide angularly robust filtering when mounted on the end of a fibre facet. This takes forward the lab-on-fibre concept, as filtering is just an example of what can be achieved with this platform. We have also demonstrated the ability of membranes of the same material, SU8, to be manipulated in an optical tweezers system. All-optical manipulation of photonic membranes is a testbed for a range of new experiments which can be performed. Optical tweezers not only present themselves as a tool for precise positioning of photonic membranes. They, and other optical manipulation techniques, provide the means to probe those regimes where photonic function and mechanical stretching and bending interrelate. It is those regimes that we intend to investigate, using photonic membranes as our starting point.

\section{ACKNOWLEDGMENTS}

We thank Mathivanan Damodaran for contribution on the optical manipulation experiments. ADF acknowledges support from EPSRC (EP/I004602/1 and EP/J004200/1).

\section{REFERENCES}

[1] Zhou, L., Wanga, A., Wu, S.-C., Sun, J., Park, S., and Jackson, T. N., "All-organic active matrix flexible display," Appl. Phys. Lett. 88(8), 083502 (2006). 
[2] Siegel, A. C., Phillips, S. T., Wiley, B. J., and Whitesides, G. M., "Thin, lightweight, foldable thermochromic displays on paper.," Lab Chip 9, 2775-81 (Oct. 2009).

[3] Yoon, J., Li, L., Semichaevsky, A. V., Ryu, J. H., Johnson, H. T., Nuzzo, R. G., and Rogers, J. A., "Flexible concentrator photovoltaics based on microscale silicon solar cells embedded in luminescent waveguides.," Nat. Commun. 2, 343 (Jan. 2011).

[4] Aksu, S., Huang, M., Artar, A., Yanik, A. A., Selvarasah, S., Dokmeci, M. R., and Altug, H., "Flexible plasmonics on unconventional and nonplanar substrates.," Adv. Mater. 23, 4422-30 (Oct. 2011).

[5] Li, G. X., Chen, S. M., Wong, W. H., Pun, E. Y. B., and Cheah, K. W., "Highly flexible near-infrared metamaterials," Opt. Express 20, 397 (Dec. 2011).

[6] Pryce, I. M., Aydin, K., Kelaita, Y. A., Briggs, R. M., and Atwater, H. A., "Highly strained compliant optical metamaterials with large frequency tunability.," Nano Lett. 10, 4222-4227 (Oct. 2010).

[7] Smythe, E. J., Dickey, M. D., Whitesides, G. M., and Capasso, F., "A technique to transfer metallic nanoscale patterns to small and non-planar surfaces.," ACS Nano 3, 59-65 (Jan. 2009).

[8] Engheta, N., "Thin Absorbing Screens Using Metamaterial Surfaces," in Antennas Propag. Soc. Int. Symp. IEEE, 392-395 (2002).

[9] Alù, A. and Engheta, N., "Optical wave interaction with two-dimensional arrays of plasmonic nanoparticles," in Structured Surfaces as Opt. Metamaterials, Maradudin, A. A., ed., ch. 3, 58-93, Cambridge University Press (2011).

[10] Di Falco, A., Ploschner, M., and Krauss, T. F., "Flexible metamaterials at visible wavelengths," New J. Phys. 12, 113006 (Nov. 2010).

[11] Dolling, G., Enkrich, C., Wegener, M., Soukoulis, C. M., and Linden, S., "Simultaneous negative phase and group velocity of light in a metamaterial.," Science 312, 892-4 (May 2006).

[12] Di Falco, A., Zhao, Y., and Alù, A., "Optical metasurfaces with robust angular response on flexible substrates," Appl. Phys. Lett. 99(16), 163110 (2011).

[13] Dao, M., Lim, C., and Suresh, S., "Mechanics of the human red blood cell deformed by optical tweezers," J. Mech. Phys. Solids 51, 2259-2280 (Nov. 2003).

[14] Kolle, M., Zheng, B., Gibbons, N., Baumberg, J. J., and Steiner, U., "Stretch-tuneable dielectric mirrors and optical microcavities," Opt. Express 18, 4356-4364 (Feb. 2010).

[15] Beyer, A., Godt, A., Amin, I., Nottbohm, C. T., Schmidt, C., Zhao, J., and Gölzhäuser, A., "Fully crosslinked and chemically patterned self-assembled monolayers.," Phys. Chem. Chem. Phys. 10, 7233-8 (Dec. 2008).

[16] She, Z., Di Falco, A., Hähner, G., and Buck, M., "Electron-beam patterned self-assembled monolayers as templates for $\mathrm{Cu}$ electrodeposition and lift-off.," Beilstein J. Nanotechnol. 3, 101-13 (Jan. 2012).

[17] Blackley, C., Drummond, L., Fikouras, A., André, P., and Di Falco, A., "Hybrid Bottom-up Approach for Flexible Plasmonics," in PECSX 10th Int. Symp. Photonic Electromagn. Cryst. Struct., 1(d) (2012).

[18] Consales, M., Ricciardi, A., Crescitelli, A., Esposito, E., Cutolo, A., and Cusano, A., "Lab-on-fiber technology: toward multifunctional optical nanoprobes.," ACS Nano 6, 3163-3170 (Apr. 2012).

[19] Shambat, G., Provine, J., Rivoire, K., Sarmiento, T., Harris, J., and Vuckovic, J., "Optical fiber tips functionalized with semiconductor photonic crystal cavities," Appl. Phys. Lett. 99(19), 191102 (2011).

[20] Reader-Harris, P., Ricciardi, A., Krauss, T. F., and Di Falco, A., "Optical guided mode resonance filter on a flexible substrate," Opt. Express 21(1), 1002-1007 (2013).

[21] Magnusson, R. and Wang, S. S., "New principle for optical filters," Appl. Phys. Lett. 61(9), 1022-1024 (1992).

[22] Moharam, M. G., Grann, E. B., Pommet, D. A., and Gaylord, T. K., "Formulation for stable and efficient implementation of the rigorous coupled-wave analysis of binary gratings," J. Opt. Soc. Am. A 12, 1068-1076 (May 1995).

[23] Fehrembach, A.-L., Talneau, A., Boyko, O., Lemarchand, F., and Sentenac, A., "Experimental demonstration of a narrowband, angular tolerant, polarization independent, doubly periodic resonant grating filter.," Opt. Lett. 32, 2269-2271 (Aug. 2007).

[24] Sakat, E., Héron, S., Bouchon, P., and Vincent, G., "Metaldielectric bi-atomic structure for angular-tolerant spectral filtering," Opt. Lett. 38(4), 425-427 (2013). 
[25] Galajda, P. and Ormos, P., "Orientation of flat particles in optical tweezers by linearly polarized light.," Opt. Express 11, 446-451 (2003).

[26] Reicherter, M., Haist, T., Wagemann, E. U., and Tiziani, H. J., "Optical particle trapping with computergenerated holograms written on a liquid-crystal display.," Opt. Lett. 24, 608-10 (May 1999).

[27] Čižmár, T., Mazilu, M., and Dholakia, K., "In situ wavefront correction and its application to micromanipulation," Nat. Photonics 4(May), 388 - 394 (2010). 